\title{
Consistent Cache Maintenance for Database Driven Websites
}

\author{
Paweł Leszczyński and Krzysztof Stencel \\ Faculty of Mathematics and Computer Science \\ Nicolaus Copernicus University \\ Chopina 12/18, 87-100 Toruń \\ \{pawel.leszczynski, stencel\}@mat.umk.pl \\ http://www.mat.umk.pl
}

\begin{abstract}
Since databases became bottlenecks of modern web applications, several techniques of caching data have been proposed. Caching data helps to resolve a problem of a database scalability, however it introduces an additional problem of a consistency maintenance. We are going to develop an existing caching model for an automatic consistency maintenance of the cached data and data stored in a database. We prepare for proposing a dependency graph which provides a mapper between update statements in a relational database and cached objects. When an update on a database is performed the graph allows detecting cached objects which have to be invalidated in order to preserve the consistency of the cache and the data source. We believe that the constructed model allows keeping the number of invalidations as low as possible. We also provide some benchmarks which prove that our method is efficient when compared with other approaches.
\end{abstract}

Keywords: web applications, scalability, database caching, cache consistency.

\section{Introduction}

WEB 2.0 applications are data-intensive. As the number of users grows, the backend database rapidly becomes a bottleneck. Thus, various data caching techniques have been developed. Most e-commerce applications have high browseto-buy ratios [6], which means that the read operations are dominant. We are going to develop a novel method of running the cache of a data-intensive WEB 2.0 application. The goal of our research is to minimize the number of objects residing in a cache which must be invalidated after an update to the stored data.

The problem of maintaining cached data up to date is difficult and known for a long time from a materialized view maintenance. However we are going to solve it for WEB 2.0 applications which allows us to define additional model assumptions. As stated earlier read operations are dominant. We remark that web applications use only basic SQL statements and we can limit SQL syntax complexity in our model. We can also identify most of performed statements

F. Daniel and F.M. Facca (Eds.): ICWE 2010 Workshops, LNCS 6385, pp. 576-580, 2010.

(C) Springer-Verlag Berlin Heidelberg 2010 
before an application deployment and find the frequent ones to cache them and improve performance. These assumptions clearly simplify the problem and give a chance of solving it although general solution may be still unknown.

\section{Motivating Example-A Community Forum Application}

Let us now consider a community forum application as an example. From the database's point of view the website consists of three views which are database extensive: listing forums, listing topics and listing posts. Figure 1 contains a view of a real forum application to show which data is needed when displaying list of topics.

\begin{tabular}{|l|c|l|}
\hline TOPICS & REPLIES & LAST POST \\
\hline $\begin{array}{l}\text { [Tutorial] How to install } \\
\text { by batt - Sat Jan 26, } 2008 \text { 3:36 am }\end{array}$ & 20 & $\begin{array}{l}\text { by g_tech9 [a } \\
\text { Wed Mar 04, 20096:15 am }\end{array}$ \\
\hline
\end{tabular}

Fig. 1. The figure shows a single line from a list of visible topics. Each line contains: a topic name which is the first post name, an owner of the topic and the date it was created, a post count, and an information about the last post.

Performing a query to display it each time the website is loaded is too extensive when avoiding a redundancy in the database. This can be done by providing extra counters, an information about the last post, etc. However this introduces problem of maintaining counters by an application logic and does not scale well.

The solution is to use a cache objects in memory. As on the figure 11 each object can contain topic data, post counter and information about the last post added. However, the data is updated by the users who add new posts. Whenever this happens, many post counters have to be recalculated. The desired property of a cache is to recompute only those counters which have become invalid and possibly nothing else. The idea of caching objects and storing them in a keyvalue storage is widely applied by mechanisms like: Memcached [12, Velocity [13] or Redis [14. However all of them lack automatic consistency maintenance and require the programmer to take care of cached data to be up to date.

In this paper we show a novel solution to the problem of consistent cache for WEB 2.0 applications. As the result, of this research, web engineers will get a scalable web cache which fully prevents storing stale data.

\section{Related Work}

Before the WEB 2.0 era several techniques of caching whole websites have been proposed and surveyed in 1115. They construct mappers between URLs and database statements. However this approach loses efficiency in WEB 2.0 applications since the same HTML can used on many pages and it does not make sense to invalidate the whole pages. 
When caching single queries it is be hard to discover similarities and differences between the queries and their result, for example when they return the same result in a different order. The other option is storing table fragments, which can be understood as storing data sets in caches and allowing them to be queried [5616]. This however lacks count operations which are nowadays common.

Authors of 2:34 present model based on statements' templates which is similar to ours. However they cannot handle join or aggregation in select statements. Our approach is based on a graph which edges determine the impact of the update statements on the cached data. The idea of the graph representation has been first presented in [7/89]. The vertexes of the graph represent instances of update statements and cached data objects. However nowadays most webpages are personalized and number of data objects has increased and multiplied by a number of application users. According to this the graph size grows rapidly and the method becomes impractical. In our approach vertexes of the dependency graph represent classes of statements instead of instances, which reduces the number of vertexes.

\section{Research}

According to the Brewer's theorem [1] it is impossible to provide consistency, availability and partition tolerance at once in a single system. A distributed system can satisfy any two of these guarantees at the same time, but not all three. Our research can be defined as finding the best trade-off between those three parameters in the terms of WEB 2.0 applications.

The planned research is divided into two parts. First an algorithm for maintaining data consistency needs to be developed and then tested in multiple benchmarks to evaluate its efficiency. The first part is mainly accomplished. We construct the dependency graph between update statements and select statements used for creating cached objects. Our construction does not use concrete statements but groups them into classes. This is efficient since in WEB 2.0 era the same statements are executed several times having only different parameters. This allows us to reduce a number of graph vertexes.

An example of the dependency graph for a simple forum application can be seen on a figure 2. The graph consists of vertexes of update statements $U_{1}, U_{2}, \ldots$, vertexes of database columns in tables, vertexes of select statements $S_{1}, S_{2}, \ldots$ and vertex of cached object topic created by them. When an update is performed we check which object vertexes may need invalidation and then resolve object instances which are not up to date. The graph edges determine dependencies between vertexes and let us trace the impact of an update statement.

The second part of the research involves providing plausible results of the constructed model carried out in different benchmarks: RUBiS [17, RUBBoS 18 and TPC-W 19. These all provide us with web application and client emulator which visits websites and performs operation according to a predefined transition probabilities. 
We are going to examine applications in different modes: without caching, caching with a defined time-to-live of the cached objects and caching with the invalidation management based on the dependency graph.

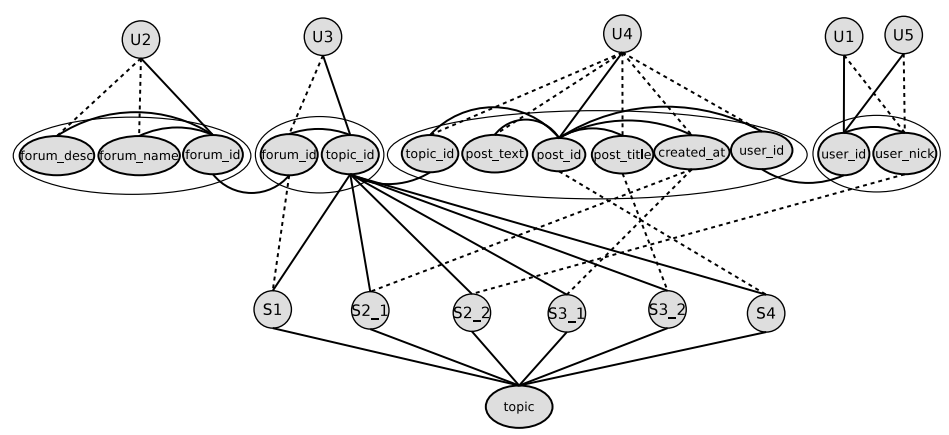

Fig. 2. A graph created for a topic objects in the community forum example

When inventing the dependency graph and invalidation algorithm we wanted to estimate the efficiency of the constructed algorithm. Therefore we have implemented the algorithm in a RUBiS benchmark. A figure 3 display achieved results. Our consistency preserving model reduces up to $54 \%$ of performed queries. This proves the presented model to be worth further development and performing more benchmarks. To do so we are heading towards creating transparent database proxy which implements our algorithm and detects statements which can be computed from the cache. The construction of the proxy gives us the possibility to carry out experiments on different benchmarks since it does not involve any changes in an application.

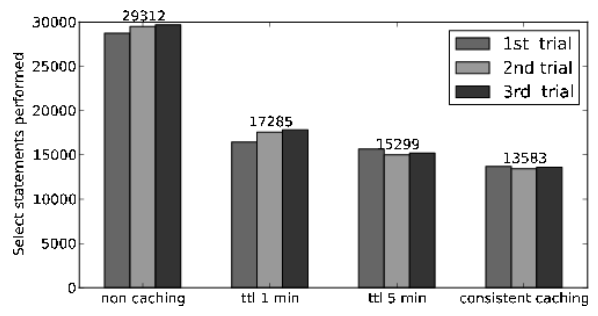

Fig. 3. The comparison of different caching techniques. The numbers indicate average count of select statements for each technique.

Additionally, when compared to materialized views, our model does not assume anything of the data stored in cached objects. As a result it allows to contain HTML code fragments. The research can be extended to providing intelligent mapper between SQL statements and HTML code fragments generated 
according to them. Such a mapper is strongly desired in modern WEB 2.0 applications where website consists of many separate components displayed under different url addresses.

\section{References}

1. Brewer, E.A.: Towards robust distributed systems. In: Annual ACM Symposium on Principles of Distributed Computing (2000)

2. Garrod, C., Manjhi, A., Ailamaki, A., Maggs, B., Mowry, T., Olston, C., Tomasic, A.: Scalable query result caching for web applications. Proceedings of the VLDB Endowment archive 1(1), 550-561 (2008)

3. Garrod, C., Manjhi, A., Ailamaki, A., Maggs, B., Mowry, T., Olston, C., Tomasic, A.: Scalable Consistency Management for Web Database Caches. Computer Science Technical Reports, School of Computer Science, Carnegie Mellon University (2006)

4. Manjhi, A., Gibbons, P.B., Ailamaki, A., Garrod, C., Maggs, B., Mowry, T.C., Olston, C., Tomasic, A., Yu, H.: Invalidation Clues for Database Scalability Services. In: Proceedings of the 23rd International Conference on Data Engineering (2006)

5. Altnel, M., Bornhvd, C., Krishnamurthy, S., Mohan, C., Pirahesh, H., Reinwald, B.: Cache tables: Paving the way for an adaptive database cache. In: Proc. VLDB 2003, pp. 718-729 (2003)

6. Luo, Q., Krishnamurthy, S., Mohan, C., Pirahesh, H., Woo, H., Lindsay, B., Naughton, J.: Middle-tier database caching for e-business. In: Proceedings of the 2002 ACM SIGMOD international conference on Management of data, pp. 600-611 (2002)

7. Iyengar, A., Challenger, J., Dias, D., Dantzig, P.: High-Performance Web Site Design Techniques. IEEE Internet Computing (4), 17-26 (2000)

8. Challenger, J., Dantzig, P., Iyengar, A., Squillante, M.S., Zhang, L.: Efficiently Serving Dynamic Data at Highly Accessed Web Sites. IEEE/ACM Transactions on Networking (12), 233-246 (2004)

9. Challenger, J., Iyengar, A., Dantzig, P.: A Scalable System for Consistently Caching Dynamic Web Data (1999)

10. Zhao, W., Schulzrinne, H.: DotSlash: Providing Dynamic Scalability to Web Applications with On-demand Distributed Query Result Caching, Computer Science Technical Reports, Columbia University (2005)

11. Katsaros, D., Manolopoulos, Y.: Cache management for Web-powered databases. Web-Powered Databases, 201-242 (2002)

12. Memcached, Danga Interactive, http://www.danga.com/memcached/

13. Velocity, http://code.msdn.microsoft.com/velocity

14. Redis, http://code.google.com/p/redis/

15. Li, W., et al.: CachePortal II: Acceleration of very large scale data center-hosted database-driven web applications. In: Proc. VLDB (2003)

16. Amiri, K., Tewari, R.: DBProxy: A Dynamic Data Cache for Web Applications. In: Proc. ICDE, pp. 821-831 (2003)

17. RUBiS (Rice University Bidding System), http://rubis.ow2.org/

18. RUBBoS (Bulletin Board Benchmark), http://jmob.ow2.org/rubbos.html

19. TPC-W (Transactional Web e-Commerce benchmark), http://www.tpc.org 